\title{
Working
}

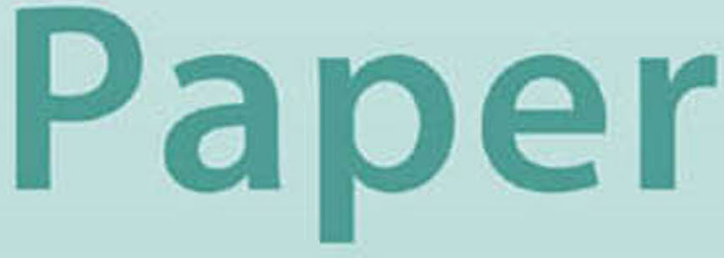




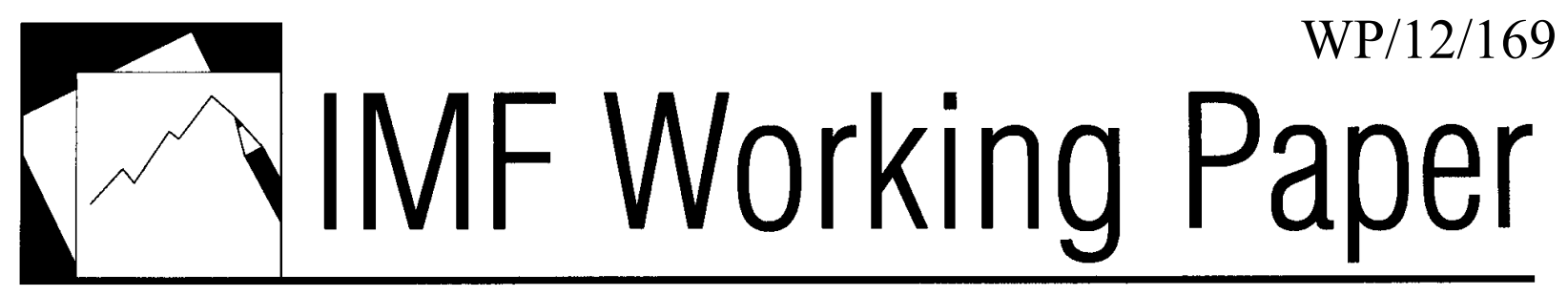

On the Extrapolation with the Denton Proportional Benchmarking Method

Tommaso Di Fonzo and Marco Marini 


\title{
IMF Working Paper
}

Statistics Department

\section{On the Extrapolation with the Denton Proportional Benchmarking Method}

\section{Prepared by Tommaso Di Fonzo and Marco Marini ${ }^{1}$}

Authorized for distribution by Kim Zieschang

June 2012

\section{This Working Paper should not be reported as representing the views of the IMF.}

The views expressed in this Working Paper are those of the author(s) and do not necessarily represent those of the IMF or IMF policy. Working Papers describe research in progress by the author(s) and are published to elicit comments and to further debate.

\begin{abstract}
Statistical offices have often recourse to benchmarking methods for compiling quarterly national accounts (QNA). Benchmarking methods employ quarterly indicator series (i) to distribute annual, more reliable series of national accounts and (ii) to extrapolate the most recent quarters not yet covered by annual benchmarks. The Proportional First Differences (PFD) benchmarking method proposed by Denton (1971) is a widely used solution for distribution, but in extrapolation it may suffer when the movements in the indicator series do not match consistently the movements in the target annual benchmarks. For this reason, an enhanced formula for extrapolation was recommended by the IMF's Quarterly National Accounts Manual: Concepts, Data Sources, and Compilation (2001). We discuss the rationale behind this technique, and propose a matrix formulation of it. In addition, we present applications of the enhanced formula to artificial and real-life benchmarking examples showing how the extrapolations for the most recent quarters can be improved.
\end{abstract}

JEL Classification Numbers: C22, C61, C82

Keywords: Benchmarking, Movement preservation, Extrapolation.

Authors’ E-Mail Addresses: tommaso.difonzo@istat.it; mmarini@imf.org.

\footnotetext{
${ }^{1}$ We would like to thank Kim Zieschang and Ethan Weisman (IMF's Statistics Department) for their helpful comments on this paper.
} 
Abstract

I. Introduction $\underline{3}$

II. The Denton PFD Benchmarking Method ............................................................ 4

III. The Enhanced Denton PFD Method for Extrapolation..................................................

A. An Approximation of the Enhanced PFD Method ............................................... 7

IV. An Example with Artificial Data ..................................................................... 9

V. An Application to Real-Life Data.....................................................................14

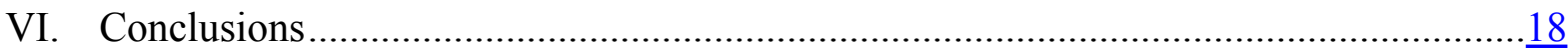

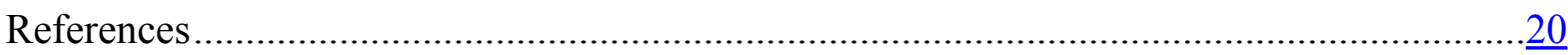

\section{Tables}

1. Extrapolation Using Forecast BI Ratios (Example 6.2, QNA Manual, 2001)..................10

2. Enhanced Denton PFD: Comparison Between the Shortcut and the Analytical Solution.. $\underline{12}$

3. Enhanced Denton PFD: Comparison with the Indicator Series.................................12

4. Basic Denton PFD vs. Enhanced Denton PFD: MSD of Quarterly Growth Rates............. 12

5. Enhanced Denton PFD: Comparison Between the Analytical Solution and the Shortcut Version with Different BI Ratios ...................................................................................

6. Forecasting Manufacturing Value Added in 2009 Using IPI: a Comparison Between PFD and EPFD. 


\section{INTRODUCTION}

Benchmarking techniques are typically used in national accounts to derive quarterly estimates of an annual aggregate. The benchmarking problem can be split into two parts: (i) distribution and (ii) extrapolation. In the former case the procedure is used to generate quarterly data which are both consistent with annual data (i.e., the sum of the quarters is equal to the annual level) and as close as possible to the movements of a quarterly preliminary series. Extrapolation refers to the calculation of quarterly forecasts of the target variable according to the movements in the quarterly preliminary series before the annual benchmark is available.

To avoid steps between consecutive years, benchmarking techniques based on some movement preservation principles are recommended. A widely used benchmarking method is the one proposed by Denton (1971), with the modification for the starting condition provided by Cholette (1984). This procedure considers an objective function according to which the proportional period-to-period changes (or Proportional First Differences (PFD)) of the benchmarked series be as close as possible to those of the preliminary figures. Hereafter, this procedure will be referred to as the basic Denton PFD benchmarking method.

Under the given constraints, the Denton PFD technique is aimed at preserving at the best $^{2}$ the movements in the preliminary series. When it comes to extrapolation, the results from this procedure may not be fully satisfactory. This problem is outlined in the IMF's Quarterly National Accounts Manual: Concepts, Data Sources, and Compilation (Bloem et al., 2001, hereafter the QNA Manual). To avoid a possible bias and improve the quality of the estimates in extrapolation, the QNA Manual proposes an enhanced version of the Denton PFD solution. Such enhancement is based on a forecast of the annual benchmark-to-indicator (BI) ratio for the next year given by the user, so that the quarterly extrapolations are in line with that annual BI forecast.

The QNA Manual proposes the objective criterion for the enhanced Denton PFD benchmarking method. However, it does not offer a matrix formulation of the problem from which an analytical solution can be derived. A shortcut version is instead presented, which turns out to give "similar results for less volatile series" (p. 93). In this paper we formalize the problem solved by the enhanced Denton PFD method in matrix terms, derive the analytical solution, and compare the results obtained from it to both the shortcut version and the basic Denton formula using artificial and real-life examples. ${ }^{3}$

\footnotetext{
${ }^{2}$ The Denton PFD method is a good approximation of the ideal movement preservation principle, which is based on the period-to-period rates of the variables involved (see QNA manual, p. 6.A1.12). This benchmarking problem is nonlinear and can be solved using optimization procedures, as discussed by Di Fonzo and Marini (2011).

${ }^{3}$ The analytical solution of the enhanced Denton method has been already proposed in the related work by Di Fonzo and Marini (2009).
} 
The paper is structured as follows. Section II presents the basic Denton PFD benchmarking method. Section III provides the matrix formulation of the enhanced solution and briefly discusses the shortcut version suggested in the QNA Manual. The basic formula and the enhanced formula are then compared using applications with artificial data and real-life data (Section IV and V). Some conclusions are drawn in the final section.

\section{The Denton PFD Benchmarking Method}

Let us denote with $\mathbf{y}=\left(y_{1}, \ldots, y_{s N}\right)$ the $(s N \times 1)$ vector of unknown values to be estimated, and with $\mathbf{y}_{0}=\left(y_{0,1}, \ldots, y_{0, N}\right)$ the $(N \times 1)$ vector of known aggregated values. We assume that the two vectors are linked by a known temporal aggregation relationship

$$
\mathbf{J y}=\mathbf{y}_{0},
$$

where $\mathbf{J}$ is the $(N \times s N)$ temporal aggregation matrix ${ }^{4}$

$$
\mathbf{J}=\mathbf{I}_{N} \otimes \mathbf{1}_{s}^{\prime}
$$

and $s$ the aggregation order (for example, $s=4$ for quarterly-to-annual aggregation). Let us define $\mathbf{p}=\left(p_{1}, \ldots, p_{s N}\right)$ the $(s N \times 1)$ vector of preliminary values for which

$$
\mathbf{J p} \neq \mathbf{y}_{0} .
$$

The Denton PFD benchmarking method looks for values in $\mathbf{y}$ whose movements are as close as possible to the values of the preliminary series ${ }^{5} \mathbf{p}$ and, at the same time, satisfy constraint (1). Based on this original proposal by Denton (1971), Cholette (1984) provides the solution to a modified ${ }^{6}$ constrained quadratic minimization problem according to which the proportionate difference between the benchmarked series and the preliminary series must be as constant as possible:

\footnotetext{
${ }^{4}$ For the sake of clarity, in this paper we only consider the case of temporal aggregation by sum.

${ }^{5}$ The QNA manual refers to the preliminary series as the indicator series. In our opinion, however, the concept of indicator is better suited in a temporal disaggregation framework where a statistical/econometric relationship is established between the related series (i.e., the indicator) and the variable to be estimated. However, in this paper we will use both terms with the same meaning.

${ }^{6}$ The original version of Denton (1971) imposes the constraint $y_{0}=p_{0}$ and includes the first correction term $y_{1}=p_{1}$ in the objective criterion. The modified variant by Cholette (1984) omits the first term.
} 


$$
\begin{aligned}
& \min _{y_{t}} \sum_{t=2}^{s N}\left(\frac{y_{t}}{p_{t}}-\frac{y_{t-1}}{p_{t-1}}\right)^{2} \\
& \text { s.t. } \quad \sum_{t \in T} y_{t}=y_{0, T} \quad \text { for } T=1, \ldots, N .
\end{aligned}
$$

In matrix notation, problem (2) can be written as

$$
\begin{aligned}
& \min _{\mathbf{y}}(\mathbf{y}-\mathbf{p})^{\prime} \mathbf{Q}(\mathbf{y}-\mathbf{p}) \\
& \text { s.t. } \quad \mathbf{J y}=\mathbf{y}_{0}
\end{aligned}
$$

with

$$
\mathbf{Q}=\hat{\mathbf{p}}^{-1} \mathbf{D}^{\prime} \mathbf{D} \hat{\mathbf{p}}^{-1}
$$

$\hat{\mathbf{p}}=\operatorname{diag}(\mathbf{p})$, and $\mathbf{D}$ the $(s N-1 \times s N)$ first differences matrix

$$
\mathbf{D}=\left[\begin{array}{lllll}
-1 & 1 & 0 & \ldots & 0 \\
0 & -1 & 1 & \ldots & 0 \\
\vdots & \vdots & \vdots & \ddots & \vdots \\
0 & 0 & 0 & \ldots & 1
\end{array}\right]
$$

After some algebra, Di Fonzo (2003) provides the following simplified solution to constrained optimization problem (3):

$$
\left[\begin{array}{l}
\mathbf{y} \\
\boldsymbol{\lambda}
\end{array}\right]=\left[\begin{array}{cc}
\hat{\mathbf{p}} & \mathbf{0} \\
\mathbf{0} & \mathbf{I}_{s N}
\end{array}\right]\left[\begin{array}{cc}
\mathbf{D}^{\prime} \mathbf{D} & \hat{\mathbf{p}} \mathbf{J}^{\prime} \\
\mathbf{J} \hat{\mathbf{p}} & \mathbf{0}
\end{array}\right]^{-1}\left[\begin{array}{c}
\mathbf{0} \\
\mathbf{y}_{0}
\end{array}\right]
$$

The benchmarking solution (4) can also be used for extrapolation. Suppose $k$ additional preliminary observations are available for the most recent high-frequency periods (say, quarters), with $k=1, \ldots, s^{7}$. The length of the preliminary vector $\mathbf{p}$ will be $n=s N+k$. To obtain the extrapolated values $y_{t}$ for $t=s N+1, \ldots, s N+k$, the aggregation matrix $\mathbf{J}$ needs to be extended with $k$ zero columns

$$
\mathbf{J}=\left[\mathbf{I}_{N} \otimes \mathbf{1}_{s}^{\prime} \quad \mathbf{0}_{N \times k}\right]
$$

and formula (4) can be applied. The form of $\mathbf{J}$ implies that for all extrapolated periods the BI ratio corresponds to the $\mathrm{BI}$ ratio of the fourth quarter covered by the last benchmark year, that is $y_{s N} / p_{s N}$ (see QNA Manual, p. 88). This implies that the quarterly growth rates of $y_{t}$ in

\footnotetext{
${ }^{7}$ The problem can easily be extended to deal with extrapolation for periods longer than one year.
} 
the extrapolated periods will correspond to the quarterly growth rates of $p_{t}$; however, since the annual BI ratio for the next year will likely be different from the annual BI ratio of the previous year, the annual growth rate $y_{0, N+1} / y_{0, N}$ will be different from the annual growth rate of the indicator series, $p_{0, N+1} / p_{0, N}$ (with $p_{0 T}$ the annual aggregation of the quarterly preliminary series).

This characteristic of the Denton PFD formula in extrapolation can be unsatisfactory for the user. First, this method does not take into account the historical relationship between the indicator series and the target variable. If, for example, the growth in the indicator constantly underestimates the growth in the target variable, one may want to see this bias reflected in the extrapolated quarters so that the growth in the target variable is lower than the growth in the indicator series. Second, the annual growth rate of the target variable is implicitly derived from the extrapolation of the last available BI ratio. The user exercises no control over this, because the extrapolation is purely mechanical. A method which offers more control in extrapolation with the Denton formula is presented in the next section.

\section{The Enhanced Denton PFD Method FOR EXTRAPOlation}

The QNA Manual proposes a modification to the basic Denton PFD method that allows introducing an explicit forecast of the BI ratio for the next year. Starting from the same constrained problem in (3), and assuming $s=4$, the following additional constraint is added

$$
\sum_{t=s N+1}^{s(N+1)} \frac{y_{t}}{p_{t}} w_{t-4}=b_{N+1}
$$

where

$$
w_{t}=\frac{p_{t}}{p_{0, T}}, \quad \text { with } T=\left[\frac{t-1}{s}\right]+1,
$$

where $[a]$ is the integer part of the real number $a$. The weight $w_{t}$ is the share of each subperiod with respect to the relevant yearly aggregated preliminary series, and

$$
b_{N+1}=\frac{y_{0, N+1}}{p_{0, N+1}} .
$$

Of course the BI ratio $b_{N+1}$ is not known at the time of the extrapolation for the quarters of the year $N+1$, therefore a forecast is required. For this purpose, it is often convenient to relate $b_{N+1}$ to the last available $\mathrm{BI}$ ratio, $b_{N}$, as follows

$$
b_{N+1}=\frac{y_{0, N}}{p_{0, N}} q
$$

where $q$ is the expected forecast change of the BI ratio for the year $N+1$. 
In matrix notation, constraint (5) can be expressed as:

$$
\left[\mathbf{R}^{-1} \hat{\mathbf{p}}_{E} \hat{\mathbf{p}}_{0 E}^{-1}\right] \mathbf{y}=b_{N+1},
$$

where

$$
\begin{aligned}
\mathbf{R} & =\left[\begin{array}{ll}
\mathbf{0}_{s N}^{\prime} & \mathbf{1}_{k}^{\prime}
\end{array}\right] \\
\hat{\mathbf{p}}^{-1} & =\operatorname{diag}\left(\mathbf{p}^{-1}\right)=\left[\begin{array}{llll}
p_{1}^{-1} & 0 & \ldots & 0 \\
0 & p_{2}^{-1} & \ldots & 0 \\
0 & 0 & \ldots & p_{n}^{-1}
\end{array}\right] \\
\hat{\mathbf{p}}_{E} & =\operatorname{diag}(\mathbf{E p})=\left[\begin{array}{lllll}
0 & 0 & \ldots & 0 & 0 \\
0 & 0 & \ldots & 0 & 0 \\
0 & 0 & \ldots & p_{n-s+1} & 0 \\
0 & 0 & \ldots & 0 & p_{n-s}
\end{array}\right] \\
\mathbf{E} & =\left[\begin{array}{ll}
\mathbf{0}_{s \times n-s} & \mathbf{0}_{s \times s} \\
\mathbf{I}_{n-s} & \mathbf{0}_{n-s \times s}
\end{array}\right] \\
\hat{\mathbf{p}}_{0 E}^{-1} & =\operatorname{diag}\left(\mathbf{E p}_{0}^{*-1}\right) \\
\mathbf{p}_{0}^{*} & =\left[\begin{array}{c}
\mathbf{p}_{0} \otimes \mathbf{1}_{s} \\
\mathbf{1}_{k}
\end{array}\right] .
\end{aligned}
$$

Denoting

$$
\mathbf{k}=\left[\mathbf{R} \hat{\mathbf{p}}^{-1} \hat{\mathbf{p}}_{E} \hat{\mathbf{p}}_{0 E}^{-1}\right]
$$

the $n$-dimensional row vector in (9), the system of constraints satisfied by the enhanced Denton PFD method for extrapolation is

$$
\begin{aligned}
{\left[\begin{array}{l}
\mathbf{J} \\
\mathbf{k}
\end{array}\right] \mathbf{y} } & =\left[\begin{array}{c}
\mathbf{y}_{0} \\
b_{N+1}
\end{array}\right], \\
\mathbf{J}^{*} \mathbf{y} & =\mathbf{y}_{0}^{*}
\end{aligned}
$$

Both the distributed values and the extrapolated values can be obtained with expression (4), using $\mathbf{J}^{*}$ instead of $\mathbf{J}$ and $\mathbf{y}_{0}^{*}$ instead of $\mathbf{y}_{0}$.

\section{A. An Approximation of the Enhanced PFD Method}

A shortcut version of the enhanced Denton PFD method is presented in the QNA Manual for illustrative purposes $\left(\S 6.35\right.$ ). Let $q_{t}$ be the BI ratio from the basic Denton PFD method

$$
q_{t}=\frac{y_{t}}{p_{t}}, \quad t=1, \ldots, N s+k .
$$


The enhanced method explicitly requires a forecast of the annual BI ratio for year $N+1$, denoted as $b_{N+1}$ in the previous section. To avoid the step problem, it is necessary that the transition from the fourth quarter of the last year to the first quarter of the current year be as smooth as possible. Let us denote with $\eta$ the quantity

$$
\eta=\frac{1}{3}\left(q_{s N}-b_{N+1}\right)
$$

which will ensure, as shown later, that the average of the extrapolated quarterly BI ratios be approximately equal to the annual forecast of the $\mathrm{BI}$ ratio $b_{N+1}$.

This quantity is used to adjust the extrapolated quarters of the basic Denton formula. The values of the quarters of the last available year are first modified, starting from the second quarter:

$$
\begin{aligned}
q_{s N-2}^{*} & =q_{s N-2}+\frac{1}{4} \eta \\
q_{s N-1}^{*} & =q_{s N-1}+\frac{1}{4} \eta \\
q_{s N}^{*} & =q_{s N}-\frac{1}{4} \eta .
\end{aligned}
$$

Then, the following recursion is used to calculate the BI ratios for the extrapolated quarters:

$$
q_{s N+k}^{*}=q_{s N+k-1}^{*}-\eta, \quad k=1, \ldots, 4 .
$$

To understand the properties of this approximation, it is useful to aggregate the quarterly BI ratios at the annual level. Two years are involved, the last available one $(N)$ and the extrapolated one $(N+1)$. The annual average of the modified $\mathrm{BI}$ ratios for the year $N$ is given by:

$$
\frac{1}{4} \sum_{k=0}^{3} q_{s N-k}^{*}
$$

Replacing each term of the sum with the original BI ratios we have

$$
\frac{1}{4}\left[q_{s N-3}+q_{s N-2}+\frac{1}{4} \eta+q_{s N-1}+\frac{1}{4} \eta+q_{s N}-\frac{1}{2} \eta\right]
$$

that is

$$
\frac{1}{4} \sum_{k=0}^{3} q_{s N-k}
$$


Then, the original annual BI ratio is preserved; this implies that for the year $N$ the sum of the quarterly benchmarked series is equal to the annual benchmark value. We now consider the annual average of the extrapolated BI ratios for the year $N+1$

$$
\frac{1}{4} \sum_{k=0}^{3} q_{s N+k}^{*}
$$

It can be transformed into

$$
\begin{array}{r}
\frac{1}{4}\left[q_{s N}-\frac{1}{2} \eta-\eta+\right. \\
q_{s N}-\frac{1}{2} \eta-2 \eta+ \\
q_{s N}-\frac{1}{2} \eta-3 \eta+ \\
\left.q_{s N}-\frac{1}{2} \eta-4 \eta\right]
\end{array}
$$

that is equal to

$$
\frac{1}{4}\left[4 q_{s N}-12 \eta\right]
$$

Replacing $\eta$ according to expression (11) we have:

$$
\frac{1}{4}\left[4 q_{s N}-4\left(q_{s N}-b_{N+1}\right)\right]=b_{N+1} .
$$

Then, the shortcut version guarantees that the (implicit) annual extrapolated BI ratio is the one requested by the user. The shortcut version, however, provides different, suboptimal results in terms of quarter-to-quarter rates of the extrapolated quarters with respect to the results achieved with the analytical solution provided in the previous section. Such differences are investigated in the following section through an example with artificial data.

\section{AN EXAMPLe With ARTIFICIAL Data ${ }^{8}$}

We consider the data shown in tables 6.2-6.4 of the QNA Manual. Table 1 shows the indicator series in the first column for the period 1998:Q1-2000:Q4. The second column presents the annual benchmark values for the years 1998 and 1999. The annual BI ratios are

\footnotetext{
${ }^{8}$ The results of the basic formula and the enhanced formula of the Denton PFD method presented in this work are obtained through an Excel user-defined function called XLPBM, which was developed within the Real Sector Division of the IMF's Statistics Department. XLPBM was developed by the IMF under its technical assistance and training programs on national accounts statistics. The XLPBM function is available upon request from the authors.
} 
shown in the third column. The quarterly BI ratios and the benchmarked series obtained with the basic Denton PFD method follow.

According to the basic Denton PFD solution, the extrapolated quarters of year 2000 are obtained by multiplying the values of the indicator series by the last available BI ratio (10.355 in quarter 1999:Q4). The enhanced Denton PFD formula requires an explicit forecast of the annual BI ratio of the year 2000. In the example it is assumed that this ratio increases of 2.0 percent from the previous year. The BI ratio of the year 2000 is therefore 10.486 (10.280 x 1.02). According to the shortcut solution given in (11), the constant $\eta$ is equal to

$$
\eta=\frac{1}{3}(10.355-10.486)=-0.044 \text {. }
$$

Table 1. Extrapolation Using Forecast BI Ratios (Example 6.2, QNA Manual, 2001)

\begin{tabular}{|c|c|c|c|c|c|c|c|c|c|c|}
\hline & & & & & & & & \multicolumn{3}{|c|}{ Quarter-to-quarter changes } \\
\hline Quarter & Indic. & $\begin{array}{c}\text { Annual } \\
\text { data }\end{array}$ & $\begin{array}{c}\text { Annual } \\
\mathrm{BI} \\
\text { ratios }\end{array}$ & $\mathrm{Bl}$ ratios & $\begin{array}{c}\text { Basic } \\
\text { Denton } \\
\text { PFD }\end{array}$ & $\begin{array}{l}\text { Enhanced } \\
\text { BI ratios } \\
\text { (shortcut) }\end{array}$ & $\begin{array}{c}\text { Enhanced } \\
\text { PFD } \\
\text { (shortcut) }\end{array}$ & Indic. & $\begin{array}{l}\text { Basic } \\
\text { PFD }\end{array}$ & $\begin{array}{c}\text { Enhanced } \\
\text { PFD } \\
\text { (shortcut) }\end{array}$ \\
\hline 1998Q1 & 98.2 & & & 9.876 & 969.8 & 9.876 & 969.8 & & & \\
\hline 1998Q2 & 100.8 & & & 9.905 & 998.4 & 9.905 & 998.4 & 2.6 & 3.0 & 3.0 \\
\hline 1998Q3 & 102.2 & & & 9.964 & 1018.3 & 9.964 & 1018.3 & 1.4 & 2.0 & 2.0 \\
\hline 1998Q4 & 100.8 & 4000.0 & 9.950 & 10.054 & 1013.4 & 10.054 & 1013.4 & -1.4 & -0.5 & -0.5 \\
\hline 1999Q1 & 99.0 & & & 10.174 & 1007.2 & 10.174 & 1007.2 & -1.8 & -0.6 & -0.6 \\
\hline 1999Q2 & 101.6 & & & 10.264 & 1042.8 & 10.253 & 1041.7 & 2.6 & 3.5 & 3.4 \\
\hline 1999Q3 & 102.7 & & & 10.325 & 1060.3 & 10.314 & 1059.2 & 1.1 & 1.7 & 1.7 \\
\hline 1999Q4 & 101.5 & 4161.4 & 10.280 & 10.355 & 1051.0 & 10.377 & 1053.2 & -1.2 & -0.9 & -0.6 \\
\hline $2000 Q 1$ & 100.5 & & & 10.355 & 1040.6 & 10.420 & 1047.2 & -1.0 & -1.0 & -0.6 \\
\hline 2000Q2 & 103.0 & & & 10.355 & 1066.5 & 10.464 & 1077.8 & 2.5 & 2.5 & 2.9 \\
\hline 2000Q3 & 103.5 & & & 10.355 & 1071.7 & 10.508 & 1087.5 & 0.5 & 0.5 & 0.9 \\
\hline 2000Q4 & 101.5 & & 10.486 & 10.355 & 1051.0 & 10.551 & 1071.0 & -1.9 & -1.9 & -1.5 \\
\hline 2000 & & & & & & & & 0.9 & 1.6 & 2.9 \\
\hline
\end{tabular}

Using the approximation illustrated in Section III. A, $\eta$ is used to obtain the enhanced quarterly BI ratios from 1999:Q2 to 2000:Q4. The corresponding extrapolated values are given next in the table.

The last three columns in Table 1 show the quarterly growth rates of the indicator series and of the benchmarked series obtained with the basic Denton PFD method and its enhanced version using the shortcut solution. The last row shows the annual rate from the four extrapolated quarters. It can be noticed that the original Denton PFD provides an annual rate 
of 1.6 percent, while the shortcut version of the enhanced method gives 2.9 percent. Compared to the growth of 0.9 percent in the indicator series, both methods provide an upward extrapolation of the quarterly benchmarked series. In the first case, the correction factor is implicitly given by the use of the BI ratio of 1999: Q4. In the latter case, the annual $\mathrm{BI}$ ratio is explicitly defined. In fact, the difference between the (annual) rate of change of the indicator and the (annual) rate of change of the extrapolated series from the enhanced Denton PFD method is exactly 2.0 percent, the same increase of the BI ratio assumed for 2000 .

So far, we have considered the shortcut version of the enhanced method presented in the QNA Manual. We now introduce the analytical solution provided in this paper. Table 3 shows the benchmarked series using the analytical solution presented in (10).

Differently from the shortcut version, the analytical solution shows different values from the basic Denton PFD in each quarter. The quarterly growth rates are different, with a maximum discrepancy of -0.3 percent in 2000:Q4. As expected, the annual rate of change of 2000 of the two versions is the same ( 2.9 percent).

It is interesting to compare the extrapolated growth rates with those of the indicator series (in the last two columns in Table 3). For the quarters of the year 2000, the shortcut version provides growth rates that are on average 0.4 percent higher than the indicator's growth rates. The analytical solution shows a higher distance in the first quarter ( 0.5 percent $)$, while differences are smaller in the other quarters ( 0.4 percent, 0.2 percent and 0.1 percent). To summarize such differences, Table 4 shows the mean squared differences (MSD) of the three benchmarking procedures under review with respect to the indicator series (square root of the average of the squared differences between the growth rates of the indicators series and the growth rates of the benchmarked series). The MSD is calculated on the whole sample and on the extrapolated quarters only (2000: Q1-2000: Q4). The smallest MSD is achieved by the basic Denton PFD solution ( 0.5946 percent). Concerning the enhanced method, the analytical solution is slightly closer to the movements of the preliminary series ( 0.6392 percent vs. 0.6523 percent of the shortcut version). The improvement of the analytical solution is much more visible if only the differences in the extrapolated quarters are considered: 0.3312 percent vs. 0.4184 percent. In extrapolation the MSD of the basic Denton PFD solution is clearly zero, because the quarterly growth rates of the benchmarked series are, by definition, the same as those in the indicator series. 


\section{Table 2. Enhanced Denton PFD: Comparison Between the Shortcut and the Analytical Solution}

\begin{tabular}{crrrrrr}
\hline & & & & \multicolumn{2}{c}{ Quarter-to-quarter changes } \\
\hline & $\begin{array}{c}\text { Enhanced } \\
\text { BI ratios } \\
\text { Quarter }\end{array}$ & $\begin{array}{c}\text { Enhanced } \\
\text { Denton PFD } \\
\text { Shortcut }\end{array}$ & $\begin{array}{c}\text { Enhanced } \\
\text { BI ratios } \\
\text { analytical }\end{array}$ & $\begin{array}{c}\text { Enhanced } \\
\text { Denton PFD } \\
\text { analytical }\end{array}$ & $\begin{array}{c}\text { Enhanced } \\
\text { Denton PFD } \\
\text { shortcut }\end{array}$ & $\begin{array}{c}\text { Enhanced } \\
\text { Denton PFD } \\
\text { Analytical }\end{array}$ \\
\hline 1998Q1 & 9.876 & 969.8 & 9.883 & 970.5 & & \\
1998Q2 & 9.905 & 998.4 & 9.909 & 998.9 & 3.0 & 2.9 \\
1998Q3 & 9.964 & 1018.3 & 9.963 & 1018.2 & 2.0 & 1.9 \\
1998Q4 & 10.054 & 1013.4 & 10.045 & 1012.5 & -0.5 & -0.6 \\
\hline 1999Q1 & 10.174 & 1007.2 & 10.153 & 1005.1 & -0.6 & -0.7 \\
1999Q2 & 10.253 & 1041.7 & 10.247 & 1041.1 & 3.4 & 3.6 \\
1999Q3 & 10.314 & 1059.2 & 10.326 & 1060.5 & 1.7 & 1.9 \\
1999Q4 & 10.377 & 1053.2 & 10.391 & 1054.7 & -0.6 & -0.5 \\
\hline 2000Q1 & 10.420 & 1047.2 & 10.441 & 1049.3 & -0.6 & -0.5 \\
2000Q2 & 10.464 & 1077.8 & 10.479 & 1079.3 & 2.9 & 2.9 \\
2000Q3 & 10.508 & 1087.5 & 10.504 & 1087.2 & 0.9 & 0.7 \\
2000Q4 & 10.551 & 1071.0 & 10.517 & 1067.5 & -1.5 & -1.8 \\
\hline 2000 & $\mathbf{1 0 . 4 8 6}$ & $\mathbf{1 0 7 0 . 9}$ & $\mathbf{1 0 . 4 8 5}$ & $\mathbf{1 0 7 0 . 8}$ & $\mathbf{2 . 9}$ & $\mathbf{2 . 9}$ \\
\hline
\end{tabular}

Table 3. Enhanced Denton PFD: Comparison with the Indicator Series

\begin{tabular}{lrrrrr}
\hline & \multicolumn{3}{c}{ Quarter-to-quarter changes } & \multicolumn{2}{c}{ Difference with indicator } \\
\hline & \multicolumn{2}{c}{$\begin{array}{c}\text { Enhanced } \\
\text { Denton PFD } \\
\text { Quarter }\end{array}$} & $\begin{array}{c}\text { Enhanced } \\
\text { Denton PFD } \\
\text { Indicator }\end{array}$ & $\begin{array}{c}\text { Enhanced } \\
\text { Denton PFD } \\
\text { shortcut }\end{array}$ & $\begin{array}{c}\text { Enhanced } \\
\text { Denton PFD } \\
\text { analytical }\end{array}$ \\
\hline 1998Q2 & 2.6 & 3.0 & 2.9 & 0.3 & 0.3 \\
1998Q3 & 1.4 & 2.0 & 1.9 & 0.6 & 0.5 \\
1998Q4 & -1.4 & -0.5 & -0.6 & 0.9 & 0.8 \\
1999Q1 & -1.8 & -0.6 & -0.7 & 1.2 & 1.1 \\
\hline 1999Q2 & 2.6 & 3.4 & 3.6 & 0.8 & 1.0 \\
1999Q3 & 1.1 & 1.7 & 1.9 & 0.6 & 0.8 \\
1999Q4 & -1.2 & -0.6 & -0.5 & 0.6 & 0.6 \\
\hline 2000Q1 & -1.0 & -0.6 & -0.5 & 0.4 & 0.5 \\
2000Q2 & 2.5 & 2.9 & 2.9 & 0.4 & 0.4 \\
2000Q3 & 0.5 & 0.9 & 0.7 & 0.4 & 0.2 \\
2000Q4 & -1.9 & -1.5 & -1.8 & 0.4 & 0.1 \\
\hline $\mathbf{2 0 0 0}$ & $\mathbf{0 . 9}$ & $\mathbf{2 . 9}$ & $\mathbf{2 . 9}$ & $\mathbf{2 . 0}$ & $\mathbf{2 . 0}$ \\
\hline
\end{tabular}

Table 4. Basic Denton PFD vs. Enhanced Denton PFD: MSD of Quarterly Growth Rates

\begin{tabular}{lrrr}
\hline & $\begin{array}{c}\text { Original } \\
\text { Denton PFD }\end{array}$ & $\begin{array}{c}\text { Enhanced } \\
\text { Denton PFD } \\
\text { shortcut }\end{array}$ & $\begin{array}{c}\text { Enhanced } \\
\text { Denton PFD } \\
\text { analytical }\end{array}$ \\
\hline MSD total & 0.5946 & 0.6523 & 0.6392 \\
MSA year 2000 & 0.0000 & 0.4184 & 0.3312 \\
\hline
\end{tabular}




\section{Table 5. Enhanced Denton PFD: Comparison Between the Analytical Solution and the Shortcut Version with Different BI Ratios}

\begin{tabular}{|c|c|c|c|c|c|c|}
\hline$b_{2000}$ & Method & PFD total & $\begin{array}{l}\text { PFD in } \\
2000\end{array}$ & MSD total & $\begin{array}{l}\text { MSD in } \\
2000\end{array}$ & $\begin{array}{l}\text { Annual rate } \\
\text { in } 2000\end{array}$ \\
\hline \multirow{2}{*}{0.94} & Enhanced PFD - Analytical & 0.222 & 0.132 & 1.413 & 1.823 & -5.1 \\
\hline & Enhanced PFD - Shortcut & 0.285 & 0.212 & 1.618 & 2.332 & -5.2 \\
\hline \multirow{2}{*}{0.96} & Enhanced PFD - Analytical & 0.130 & 0.065 & 1.074 & 1.267 & -3.1 \\
\hline & Enhanced PFD - Shortcut & 0.161 & 0.105 & 1.203 & 1.615 & -3.1 \\
\hline \multirow{2}{*}{0.98} & Enhanced PFD - Analytical & 0.070 & 0.022 & 0.785 & 0.723 & -1.1 \\
\hline & Enhanced PFD - Shortcut & 0.080 & 0.035 & 0.843 & 0.919 & -1.1 \\
\hline \multirow{2}{*}{1.00} & Enhanced PFD - Analytical & 0.042 & 0.002 & 0.610 & 0.191 & 0.9 \\
\hline & Enhanced PFD - Shortcut & 0.042 & 0.003 & 0.616 & 0.241 & 0.9 \\
\hline \multirow{2}{*}{1.02} & Enhanced PFD - Analytical & 0.046 & 0.005 & 0.639 & 0.331 & 2.9 \\
\hline & Enhanced PFD - Shortcut & 0.048 & 0.008 & 0.652 & 0.418 & 2.9 \\
\hline \multirow{2}{*}{1.04} & Enhanced PFD - Analytical & 0.083 & 0.031 & 0.845 & 0.842 & 4.9 \\
\hline & Enhanced PFD - Shortcut & 0.098 & 0.050 & 0.909 & 1.061 & 5.0 \\
\hline \multirow{3}{*}{1.06} & Enhanced PFD - Analytical & 0.152 & 0.081 & 1.129 & 1.344 & 7.0 \\
\hline & Enhanced PFD - Shortcut & 0.191 & 0.131 & 1.248 & 1.688 & 7.0 \\
\hline & Basic Denton PFD & 0.040 & 0.000 & 0.595 & 0.000 & 1.6 \\
\hline
\end{tabular}

This comparison may be affected by the particular BI ratio used in this example. To verify this, we vary the value of $b_{2000}$ within the range $[0.94,1.06]$ (from -6 percent to +6 percent), with a step of 0.02 (+2 percent). The same indicator series is used. Table 5 displays the following statistics: in the first two columns the PFD criteria, as defined in (2); in the third and fourth columns the MSD of growth rates between the indicator series and the benchmarked series. Both statistics are calculated on the whole period and considering the extrapolated quarters of the year 2000 only. Finally, the last column shows the annual rate of change of 2000. The original Denton PFD solution is shown in the last row of the table for reference.

It can be noticed that the analytical solution always outperforms the shortcut version in terms of closeness to the movements of the indicator series. Improvements are higher as the change of the extrapolated BI ratio is far from 1.0 (no change). Second, the annual extrapolated rate of change from the two versions of the enhanced method is the same (except in two cases due to rounding errors, when $b_{2000}$ is equal to 0.94 and 1.04). Finally, the basic Denton PFD solution is the method that preserves better the movements in the indicator series at the time of the extrapolation, that is when the annual benchmark for the year $N+1$ is not yet available. However, the basic Denton PFD will converge to the enhanced solution if the annual benchmark for the year $N+1$ (once included in the benchmarking process) is in line with the annual BI ratio forecast used during the extrapolation periods. Indeed, the enhanced Denton PFD solution for extrapolation also aims at reducing future revisions of quarterly data by attempting an accurate projection of the annual $\mathrm{BI}$ ratio for the next year. 


\section{An ApPlication to REAL-Life DATA}

In this section we use an experiment with real-life data to evaluate the effectiveness of the enhanced Denton PFD formula. We consider a typical benchmarking problem of national accounts: the quarterly disaggregation of the annual series of manufacturing value added (VA) using as indicator series the quarterly industrial production index (IPI) of manufacturing. IPI is a timely indicator of industrial activity and is often considered as a good short-term proxy of VA in volume terms. For this reason many countries use IPI directly to estimate manufacturing VA in the QNA (using the so called "single deflation" approach).

In this experiment we consider VA and IPI of manufacturing for the following twelve G-20 countries: Australia, Canada, France, Germany, Indonesia, Italy, Japan, Korea, Mexico, South Africa, Turkey and the United States. Time series were extracted from the "National Accounts" and "Industry and Services" themes in the Organisation for Economic Co-operation and economic development (OECD) data warehouse, available at http://stats.oecd.org/ 9 . The sample period considered goes from 2000 through 2009 . Since the data were extracted in March 2012, the national accounts data in these years should be consolidated because they incorporate both fully revised short-term statistics and more comprehensive data from annual surveys.

Our objective is to use the Denton PFD method to derive out-of-sample extrapolation of VA for 2009. This year was characterized by a strong downturn of economic activity in most industrialized countries. Many stable relationships between short-term economic indicators and annual national accounts series were seen to break down starting from 2008, including the relationship between IPI and VA of manufacturing activity. We expect to achieve some improvements with the enhanced Denton PFD in the extrapolation of 2009 if this break in the relationship is properly addressed and taken into account.

Extrapolation from the Denton PFD formula is purely mechanical. As explained in Section II, the last available quarterly BI ratio is used to extrapolate any subsequent quarters not covered by annual benchmarks. This implies that the quarterly benchmarked estimates will show the same quarterly growth rates of the indicator series used, while the annual growth rate may differ from that shown by the indicator series. On the contrary, with the enhanced formula an input is required from the user: the forecast of the annual BI ratio for the next year given in (7). In this out-of-sample exercise, we assume not to know the BI ratio for 2009. Then, the first step of the procedure is to find an appropriate method to get an accurate forecast for 2009 given the BI ratio available up to (and including) 2008.

\footnotetext{
${ }^{9}$ The G-20 countries were chosen according to the data availability in the OECD database.
} 
The QNA Manual gives some suggestions on how to calculate a forecast of the next annual BI ratio (p. 6.31). Different alternatives are given according to the movements shown by the annual BI ratio over the observed periods. Figure 1 shows the annual BI ratio for the twelve G-20 countries considered in the years $2000-2009 .{ }^{10} \mathrm{We}$ plot the 2009 data point in the charts for reference but we assume this $\mathrm{BI}$ ratio is unknown. An increase (a decrease) of the BI ratio over time signals that VA is growing faster (slower) than IPI. A stable BI ratio around a mean denotes a good approximation of the growth rates of VA by IPI. For most countries IPI seems to underestimate the VA growth in the last ten years (Germany and Korea are the only exceptions). Most BI series show a regular pattern, while abrupt changes occur only in few cases (Korea in 2001, Turkey in 2002, Mexico in 2003, Australia in 2008). Looking at these charts up to 2008, we may conclude that the annual BI series show some form of regularity over time and can be predicted based on the past observations.

Given the low number of observations, we prefer not to use sophisticated time-series models to avoid an excessive uncertainty in parameters' estimation. Instead, we choose to derive the annual BI ratio for 2009 using an average BI ratio from the most recent years. Looking at the 2000-2008 period only, we use the following rules to calculate the annual 2009 BI forecast:

- when the BI ratio does not show a clear (upward or downward) trend but it fluctuates around a long-term average, we use the average level of the $\mathrm{BI}$ ratio observed in the last five years (2004-2008). This approach is followed for Australia, Italy and Mexico;

- when the $\mathrm{BI}$ ratio displays a clear trend movement in the latest years, we use the geometric average of the growth rates of the $\mathrm{BI}$ ratio observed in the last five years (2004-2008). This approach is followed for Canada, France, Germany, Indonesia, Japan, Korea, South Africa, Turkey and the United States.

The five-year span choice to calculate the average level of the BI ratio is a subjective choice and does not reflect any particular assumption on the relationship between IPI and VA in these countries. We looked for a simple, pragmatic forecasting method to show how the quality of the extrapolated quarters (in terms of accuracy) could be enhanced with respect to the basic Denton benchmarking method.

\footnotetext{
${ }^{10}$ The different scale of the BI ratio for each country depends on the level of the VA in volume expressed in monetary terms. The IPI is expressed as an index series with reference year $2005=100$ for all countries.
} 
Figure 1. Annual Ratio Between Value Added And Industrial Production Index of Manufacturing in Selected G-20 Countries (Period: 2000-2009)

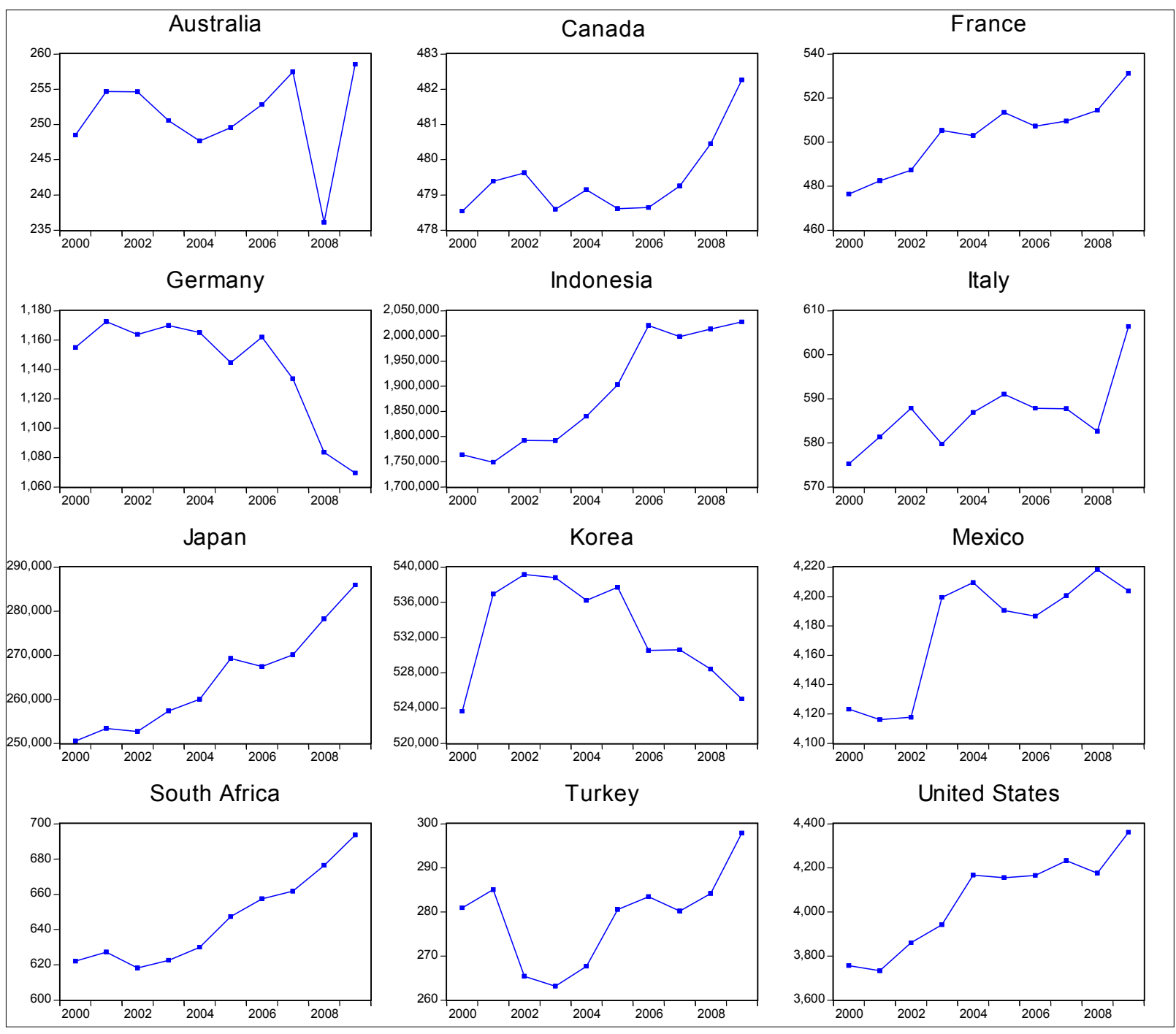

Table 6 shows the extrapolation results for 2009 using the basic formula and the enhanced formula. The first two columns contain the IPI growth and the VA growth (mostly negative, because of the global financial crisis). The IPI growth was more negative than VA growth in most countries (except Germany, Korea and Mexico). Columns in the middle of the table show the annual 2009 growth rate from the quarterly observations extrapolated with the basic Denton formula and the error with respect to the "true" observed growth rate of VA. It can be noticed that the 2009 extrapolated growth rate for VA is similar to the IPI growth rate shown in the first column, but not equal. In fact, the basic Denton PFD does not guarantee the same annual rate of the indicator series in output. More importantly, it does not take into account all the information available on the historical BI ratio movements. 


\section{Table 6. Forecasting Manufacturing Value Added in 2009 Using IPI: A Comparison Between PFD and EPFD}

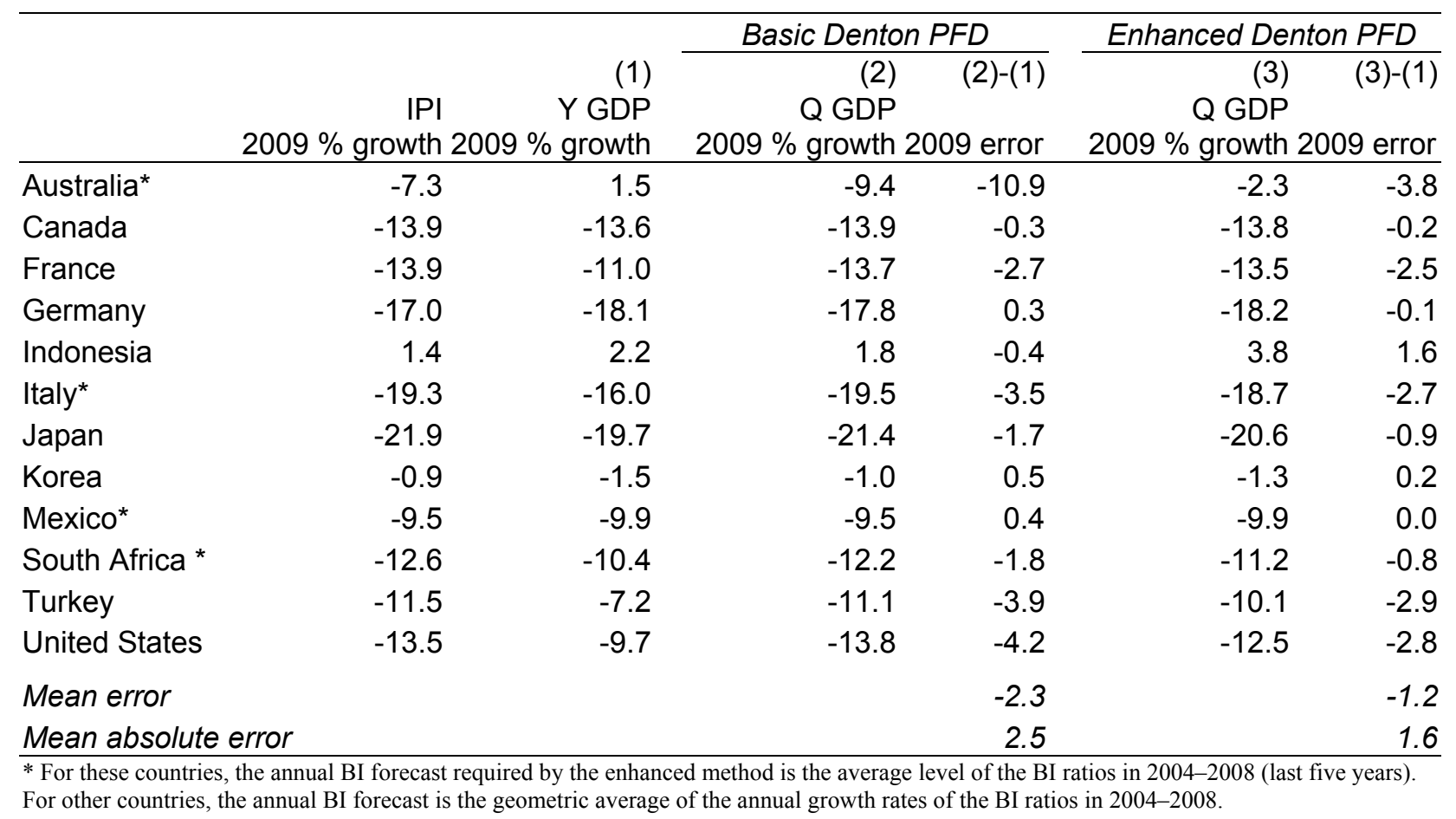

Taking into account the historical movements in the BI ratio is what the enhanced Denton formula attempts to do. The results are shown in the last two columns in Table 6. The extrapolation error is smaller (in absolute terms) for all the countries considered, with the exception of Indonesia where the enhanced method leads to an over-estimation of 1.6 percent compared to an under-estimation of only 0.4 percent with the basic formula. The enhanced formula does particularly well for Australia (an error of -3.8 percent against -10.9 percent with the basic formula), for which there was a rebound of the BI ratio in 2009 after the drop in 2008. The error with the basic Denton formula is so high because this method used the last available quarterly BI ratio (2008: Q4) to extrapolate the 2009 quarters. Since the quarterly BI in 2008: Q4 is necessarily low to stay in line with the drop in 2008 of the annual BI ratio, the extrapolated quarters are forced to remain at that low level although the average historical BI ratio is at a higher level (see top-left corner chart in Figure 1).

Overall, the mean error and the mean absolute error with the basic Denton formula are -2.3 percent and 2.5 percent. Using the enhanced formula for extrapolation, we obtain smaller average errors both in relative terms (-1.2 percent) and in absolute terms (1.6 percent).

With this example we have shown that an improvement in the forecasting accuracy of the basic Denton formula can be achieved if a good-quality annual forecast of the next year's BI ratio is used. We have also shown that, however, the enhanced Denton formula can lead to 
worse extrapolation results than the basic Denton formula when the annual forecast of the BI ratio turns out to be far from the true BI value (like for Indonesia).

\section{Conclusions}

With the basic Denton PFD method, extrapolations are calculated through a carry-forward of the last available quarterly BI ratio. The implicit annual forecast for the next year is in the end determined by the level of the last observed annual BI ratio (and, possibly, by the preceding one), which may hide a measurement bias due to preliminary information available and be unrelated to the historical relationship existing between the indicator series and the annual benchmarks. The advantage of the enhanced method proposed by the IMF's $Q N A$ Manual is that the user can explicitly introduce a forecast for the annual BI ratio of the next year based on this relationship and keep more under control the extrapolation mechanism of the benchmarking method.

This paper proposed an analytical solution, using a matrix formulation, of the enhanced Denton PFD method recommended in the QNA Manual. This solution makes it possible to implement the enhanced method through any computing software allowing matrix calculations. We showed that the shortcut version proposed in the QNA Manual guarantees the requested forecast of the current year. However, using the same example with artificial data used in the QNA Manual, we showed that the simplified recursion may distort the quarter-to-quarter movements in the indicator series. For this reason, the analytical solution presented in this paper should always be preferred when the enhanced Denton PFD benchmarking method is used.

We applied the enhanced Denton PFD formula to a typical application of benchmarking in QNA $^{11}$ (benchmarking of annual value added of manufacturing with quarterly industrial production index). We used both the basic method and the enhanced method to extrapolate manufacturing value added in 2009 for twelve G-20 countries. We calculated the required annual BI forecast for each country as the average of the BI ratio (in levels or in growth rates, according to the observed movements in the BI series) observed in the last five years of the sample period considered. We showed that using these forecasts the enhanced formula markedly improves the forecasting accuracy of the extrapolated quarters over the basic Denton PFD method.

Of course, the way in which we calculate the BI ratio forecasts in this paper proved to be effective in this particular case but cannot be generalized. However, we think that looking at

\footnotetext{
${ }^{11}$ Benchmarking techniques are not only confined to national accounts applications. The enhanced Denton method discussed in this paper may be useful in all statistical domains where high-frequency observations need to be benchmarked and extrapolated in line with more accurate low-frequency information. Government Finance Statistics (GFS) and Balance of Payment (BOP) statistics are two other examples where this problem may arise.
} 
the annual time series of $\mathrm{BI}$ ratio is always a good practice to determine if the basic Denton benchmarking method needs the proposed enhancement for extrapolation. We think that when a systematic pattern arises from the annual series of the BI ratio, especially in the latest years, the user should take advantage of this information and try to exploit it using the enhanced Denton PFD method. 


\section{REFERENCES}

Bloem, A., R. Dippelsman, and N. Mæhle, 2001, Quarterly National Accounts Manual: Concepts, Data Sources, and Compilation, (Washington, D.C. International Monetary Fund).

Cholette, P. A., 1984, “Adjusting Sub-annual Series to Yearly Benchmarks,” Survey Methodology, Vol. 10, pp. 35-49.

Dagum, E.B. and P.A. Cholette, 2006, Benchmarking, Temporal Distribution, and Reconciliation Methods for Time Series. (Springer: New York).

Denton, Frank T., 1971, “Adjustment of Monthly or Quarterly Series to Annual Totals: An Approach Based on Quadratic Minimization," Journal of the American Statistical Association, Vol. 66, pp. 99-102.

Di Fonzo, T., 2003, "Benchmarking di serie storiche economiche. Nota tecnica ed estensioni" Working paper series, N.10, (Department of Statistical Sciences, University of Padua).

Di Fonzo, T. and M. Marini, 2009, “The Enhanced Denton's Benchmarking Procedure for Extrapolation. Matrix Formulation and Practical Issues, "Working paper series, N.19, (Department of Statistical Sciences, University of Padua).

Di Fonzo, T. and M. Marini, 2011, “A Newton's Method for Benchmarking Time Series According to a Growth Rates Preservation Principle," IMF Working paper 11/179 (Washington, D.C. International Monetary Fund).

U.S. Census Bureau, X-12-ARIMA Reference manual, Version 0.3., Available via Internet: http://www.census.gov/srd/www/x12a/ 